\title{
Decade of progress in motor functional neurological disorder: continuing the momentum
}

\author{
David L Perez 이, ${ }^{1}$ Mark J Edwards, ${ }^{2}$ Glenn Nielsen, ${ }^{2}$ Kasia Kozlowska, ${ }^{3}$ \\ Mark Hallett (i), ${ }^{4}$ W Curt LaFrance, Jr (1) ${ }^{5}$
}

- Additional material is published online only. To view, please visit the journal online (http://dx.doi.org/10.1136/ jnnp-2020-323953).

${ }^{1}$ Neurology and Psychiatry, Massachusetts General Hospital, Boston, Massachusetts, USA ${ }^{2}$ Neuroscience Research Centre, St George's University of London, London, UK ${ }^{3}$ Westmead Institute of Medical Research, The Children's Hospital at Westmead, Sydney Medical School, Westmead, New South Wales, Australia

${ }^{4} \mathrm{NIH}$, NINDS, Bethesda, Maryland, USA

${ }^{5}$ Psychiatry and Neurology, Rhode Island Hospital, Providence, Rhode Island, USA

Correspondence to Dr W Curt LaFrance, Jr Psychiatry and Neurology, Rhode Island Hospital Providence, RI 2903, USA; william_lafrance_jr@brown.edu

Received 30 December 2020 Revised 8 February 2021 Accepted 23 February 2021 Published Online First 15 March 2021

Check for updates

(C) Author(s) (or their employer(s)) 2021. No commercial re-use. See rights and permissions. Published by BMJ.

To cite: Perez DL, Edwards

MJ, Nielsen G, et al. J Neurol

Neurosurg Psychiatry

2021:92:668-677.

\section{ABSTRACT}

Functional neurological disorder (FND) is a prevalent, disabling and costly condition at the neurologypsychiatry intersection. After being marginalised in the late 20th century, there has been renewed interest in this field. In this article, we review advances that have occurred over the past decade (2011-2020) across diagnosis, mechanisms, aetiologies, treatments and stigma in patients with motor FND (mFND, that is, functional movement disorder and functional limb weakness). In each content area, we also discuss the implications of recent advances and suggest future directions that will help continue the momentum of the past decade. In diagnosis, a major advance has been the emphasis on rule-in physical signs that are specific for hyperkinetic and hypokinetic functional motor symptoms. Mechanistically, greater importance has been given to determining 'how' functional neurological symptoms develop, highlighting roles for misdirected attention, expectation and self-agency, as well as abnormal influences of emotion/threat processing brain areas on motor control circuits. Aetiologically, while roles for adverse life experiences remain of interest in $\mathrm{mFND}$, there is recognition of other aetiologic contributors, and efforts are needed to investigate links between aetiological factors and mechanisms. This decade has seen the first randomised controlled trials for physiotherapy, multidisciplinary rehabilitation and psychotherapy performed in the field, with consensus recommendations for physiotherapy, occupational therapy and outcome measures also published. Across patients, clinicians, healthcare systems and society, stigma remains a major concern. While challenges persist, a patient-centred integrated clinical neuroscience approach is primed to carry forward the momentum of the past decade into the future.

\section{INTRODUCTION}

Functional neurological disorder (FND), also known as conversion disorder, is a common, disabling and costly condition at the intersection of neurology and psychiatry. ${ }^{12}$ While of interest to founding leaders across the clinical neurosciences in the late 19th century, FND was largely abandoned by academics and researchers alike during the late 20th century. ${ }^{S 1}$ The rationale for these difficulties were based in part on a Cartesian dualism of the brain and mind, limited neuropathophysiologic understanding and few evidence-based treatments. ${ }^{\text {S2 }}$ In the 21st century, a resurgence of interest in FND has occurred, catalysed by improved diagnostic specificity, an expanding 'toolbox' of treatments and new pathophysiological models that embrace patient-centred biopsychosocial formulations. ${ }^{3}$ A newly formed professional society (www.fndsociety. org), authoritative FND textbooks ${ }^{45}$ and recent special journal issues on this topic have further energised clinical and research efforts in FND.

In this narrative review, we highlight important advancements and their implications for motor FND (mFND) over the past 10 years (2011-2020) spanning functional movement disorder and functional $\operatorname{limb} /$ face weakness. We use a transdiagnostic approach across the range of functional motor symptoms given high phenotypic overlap across populations (eg, functional tremor with concurrent functional weakness in the same limb). ${ }^{\mathrm{S} 3}$ Isolated functional (psychogenic non-epileptic/dissociative) seizures, functional speech/voice disorder, functional cognitive disorder, functional sensory deficits and the spectrum of functional somatic disorders are beyond the scope of this article and have been reviewed elsewhere. ${ }^{S 4}$ Sections here detail recent developments in diagnosis, mechanisms, aetiological factors, treatments and stigma in patients with mFND. In each content area, future directions are also suggested, aimed at continuing the momentum of the past decade.

\section{DIAGNOSIS}

\section{New developments}

Establishing the diagnosis of $\mathrm{mFND}$ has been made more practicable, ${ }^{56}$ as physical examination findings with diagnostic specificity have been identified (eg, Hoover's sign with an estimated specificity of 95.7\%-99.9\%). ${ }^{6}$ Educational efforts have also made neurologists more confident in their ability to accurately diagnose patients with $\mathrm{mFND}$, discouraging extensive laboratory testing unless a comorbid neurological disorder is suspected.?

The Diagnostic and Statistical Manual of Mental Disorders-Fifth Edition (DSM-5) criteria for FND include the diagnostic features of inconsistency and incongruity on examination, emphasising positive neurological features; identifying an underlying psychological trauma has been relegated to a discussion note and removed as a criterion. ${ }^{1}$ S3 S6 Inconsistency refers to changes in manifestation over time, such as variation in tremor frequency and amplitude or remissions and exacerbations. Incongruity refers to discordance with other known neurological disorders or human anatomy and physiology. Additional general diagnostic features 
Table 1 Positive clinical features (phenotype specific) and useful adjunctive laboratory tests for the diagnosis of functional movement disorder

\begin{tabular}{|c|c|c|}
\hline $\begin{array}{l}\text { Functional motor } \\
\text { symptom }\end{array}$ & Clinical features & Laboratory tests \\
\hline Tremor & $\begin{array}{l}\text { Entrainment (tremor takes on the rhythm of paced movements performed with another } \\
\text { body part) } \\
\text { Pause with quick movement of another limb } \\
\text { Variability in frequency, amplitude } \\
\text { Tonic contraction at onset } \\
\text { Increase in amplitude with weighting } \\
\text { Coherence of tremor between two limbs } \\
\text { Whack-a-mole sign (restraint of tremor induces tremor in another body part) }\end{array}$ & $\begin{array}{l}\text { Clinical neurophysiological measurements can quantify entrainment, } \\
\text { pause with quick movement, variability, tonic contraction at onset, } \\
\text { increase amplitude with weighting and coherence between limbs }\end{array}$ \\
\hline Tic & $\begin{array}{l}\text { Lack of urge } \\
\text { Lack of voluntary control (suppressibility) }\end{array}$ & Normal Bereitschaftspotential \\
\hline Dystonia & Certain patterns such as fixed dystonia or pulling lip to one side & $\begin{array}{l}\text { Normal blink reflex recovery } \\
\text { Normal plasticity with paired associative conditioning }\end{array}$ \\
\hline Gait disorders & $\begin{array}{l}\text { Specific patterns including knee buckling, dragging a monoplegic leg, astasia-abasia, } \\
\text { excessive slowness and atypical limping } \\
\text { Better balance than claimed, including improvement with distraction } \\
\text { Either no falls, controlled falls or falling toward support } \\
\text { Chair test (can use legs to move a chair better than walking) }\end{array}$ & None \\
\hline
\end{tabular}

are distractibility and suggestibility. Most other diagnostic signs are phenotype specific and can be augmented by certain clinical neurophysiological tests. ${ }^{\mathrm{S7}}$ Clinical neurophysiological tests (eg, electromyography-assisted identification of tremor pause during ballistic movements) can either objectify bedside observations or identify features that clinicians cannot readily appreciate. ${ }^{8}$

Positive signs on examination that characterise functional limb weakness (and functional sensory and gait disorders) have been analysed for their statistical properties (including sensitivities and specificities) ${ }^{6}$ and then subjected to prospective analysis, including inter-rater reliability. ${ }^{9}$ Reliable signs of functional limb weakness include give-way/collapsing weakness, drift without pronation, cocontraction, Hoover's sign, hip abductor sign, Spinal Injuries Center Test and weakness of the sternocleidomastoid with hemiparesis.

Hyperkinetic mFND presentations include tremor, myoclonus and tics (jerky movements), dystonia, parkinsonism and gait disorders. Clinical and laboratory features are described in table 1. ${ }^{110 \mathrm{~S} 3}$ Tremor and myoclonus are readily identifiable with established clinical features and excellent neurophysiological tests that can be used for confirmation in ambiguous cases. ${ }^{11}$ S7 ${ }^{58}$ Differentiating functional versus neurogenic motor tics can be difficult if the presence of an urge or sensory tic is not present or uncertain. ${ }^{12}$ The presence of a Bereitschaftspotential (readiness potential) prior to the movement is common in functional jerky movements and is rare or foreshortened in other motor tics, but this is not definitive. ${ }^{11}$ Functional parkinsonism is usually identifiable on physical examination, but a normal dopamine transporter scan can aid the diagnosis in challenging cases. ${ }^{\text {S9 }}$ Gait disorders are usually also diagnosed by examination patterns ${ }^{913}$, perhaps the most important feature being that balance can be demonstrated to be better than what is perceived by the patient. Functional dystonia remains challenging to diagnose given some overlapping clinical features with neurogenic dystonias, and, remarkably, overlapping clinical neurophysiological features as well. ${ }^{\mathrm{S} 10}$ Fixed dystonia, ${ }^{\mathrm{S} 11}$ post-traumatic dystonia ${ }^{14}$ and intermittent lip deviation to one side ${ }^{15}$ are commonly identified functional patterns. Functional facial spasm, a common stroke mimic, is characterised by platysma hyperactivation, jaw deviation and ipsilateral eyebrow depression. ${ }^{15}$

In support of the stability of an mFND diagnosis based on examination signs, a 14-year prospective study in 76 patients with functional limb weakness showed only a $1 \%$ misdiagnosis rate. ${ }^{16}$ Notably, some patients have both functional neurological signs and other neurological conditions, such as associations with multiple sclerosis, Parkinson's disease and other neurodegenerative disorders. ${ }^{16} 17$

\section{Implications}

Improved diagnostic specificity has made it easier for neurologists to present the diagnosis to patients, which is the first step in treatment. ${ }^{18}$ It is generally necessary for the patient to agree with the diagnosis or, at least, allow for the possibility of such before moving onto additional treatments. Additionally, it has been suggested that it is helpful to demonstrate positive signs to the patient to show how the diagnosis was made. ${ }^{19}$ Increased diagnostic specificity also permits identification of cohorts with content validity for research studies.

\section{Future directions}

There is a need to further test the specificity, sensitivities and inter-rater reliability of the growing range of positive functional signs compared with other neurological populations, particularly given that statistical properties for some signs have been only tested in a single cohort. ${ }^{6}$ Additionally, functional dystonia remains among the most challenging mFND diagnoses. The overlap of clinical features and neurophysiological tests with other dystonia subtypes remains obscure and needs to be explained. However, there are some promising tests (eg, blink reflex recovery and paired-associative stimulation induced plasticity) that need further validation. ${ }^{20}$ S12 
Although relevant to only a small minority of cases, there is also a need to better distinguish mFND (where symptoms are experienced as involuntary) from factitious disorder and malingering; in both factitious disorder and malingering, there is conscious feigning of symptoms. ${ }^{21}$ The latter two presentations are rare but unfortunately influence physician attitudes toward patients, and they should be addressed differently.

Another challenge is to identify adjunctive diagnostic biomarkers. Quantitative neuroimaging alone in its current form may not provide the answer. Neuroimaging findings are valuable in understanding the neuropathophysiology of mFND, but are sufficiently subtle (and heterogeneous) that they will likely not have high sensitivity and specificity on an individual basis. While neurophysiological testing shows encouraging value in some circumstances, ${ }^{8} 11$ it will be important to show high specificity when used in relevant uncertain clinical circumstances. Composite diagnostic biomarkers across multiple neurobiological data points (electrophysiology, neuroimaging, autonomics, etc) also warrant future investigation.

Lastly, the field needs to better contextualise the overlap between mFND and other FND subtypes (eg, functional seizures), as well as to explore optimal approaches to contextualise other bodily symptoms frequently present in patients with mFND that closely relate to quality of life (eg, pain, fatigue, cognitive symptoms). ${ }^{\mathrm{S} 13 \mathrm{~S} 14}$ Furthermore, the intersection of FND, functional somatic disorders (eg, fibromyalgia) and other neuropsychiatric conditions (anxiety and traumarelated disorders, somatic symptom disorders, mild traumatic brain injury, etc) requires clarification.

\section{MECHANISMS}

\section{New developments}

An important recent focus of mechanistic theorising has been to shift the typical viewpoint from which mFND has been studied. Traditional 'Freudian' and related viewpoints have been, arguably, ones that prioritise aetiological factors over mechanisms. In other words, the precise mechanics of how a particular functional motor symptom arises has not been of high concern, and instead emphasis had been almost exclusively on the influence of hypothesised stressors and psychological factors.

Emphasis on mFND mechanisms has drawn on a broad neuroscientific knowledge base, including from the fields of motor control (eg, the underpinnings of sense of agency), cognitive-affective neuroscience (eg, attention and emotion processing) and computational neuroscience. One of the important questions such work has sought to answer is: if functional neurological symptoms are truly involuntary, what are the implicated brain mechanisms of these unconscious processes?

Research has started to coalesce around the idea that there is a mechanism (or set of inter-related mechanisms) which mediates the relationship between conscious experience of movement control and the neural networks that enable movement and related sensatory experiences to occur. In one expression of this idea grounded in the computational theory of active inference, perception and movement control rely on a dynamic relationship between actual sensory data and predictions about these data. ${ }^{22}$ The relative weighting of these 'bottom up' and 'top down' sources of information (known as precision) can be influenced by attentional focus (including modulation via limbic/salience networks). The suggestion in mFND is that abnormally strong predictions, relevant to symptoms such as weakness, tremor and gait difficulties, develop and are made more precise by body-focused attention. This drives symptom production in line with abnormal predictions and overwhelms contradictory sensory evidence. Notably, efforts are underway to test these theories, such as the recent use of the 'broken escalator' paradigm probing non-conscious and conscious forms of motor learning in patients with functional gait disorder to identify persistence of a locomotor after effect (representing a failure of deadaptation) (see figure 1). ${ }^{23}$ This mechanism can also lead to motor symptoms without a sense of agency, with functional neuroimaging studies in mFND implicating the right temporoparietal junction/inferior parietal lobule in deficits in action authorship perceptions. ${ }^{24}$

Recent work, using neuroimaging and other experimental approaches, has also begun to contextualise the role of emotion/threat processing in the pathophysiology of mFND. ${ }^{\text {S15 }}$ This research has sought to elucidate the way in which networks relevant to voluntary movement might be abnormally influenced ('hijacked') by networks serving affective and threat processing. Noteworthy findings include: (1) a direct effect of recall of relevant traumatic life events on supplementary motor area activation ${ }^{25}$; (2) abnormal connectivity between motor control areas and amygdala/insula brain areas during rest and affective provocation ${ }^{\text {S16 }}$ and (3) altered temporoparietal junction and insula cortex connectivity in the resting state. ${ }^{24} \mathrm{S16}$

\section{Implications}

An important implication of this work has been to support mFND as a brain-based condition. In this sense, it is simply a process of the mFND field catching up with the rest of neuropsychiatry, benefiting from recent neuroscientific advances, building on historical concepts, which further bridges neurology and psychiatry. However, this change has a danger of creating a solely neurocentric view of mFND, ending up swapping one extreme viewpoint (psychology only) for another (neurology only)—a sentiment that we caution against.

\section{Future directions}

The path forward is one that continues building an integrated, mechanistic framework for mFND that is neither exclusively psychological nor neurological. A key future goal is to attempt to unpack, at an individual level, the different influences on symptoms, aetiologies, treatment response and prognosis. For example, efforts to integrate active inference principles not only for sensorimotor percepts but also pertaining to interoception and 'emotion making' based on the theory of constructed emotion may provide additional mechanistic advances in mFND. ${ }^{\text {S17 }}$ How mFND neural mechanisms relate to treatment mechanisms and clinical outcomes is critically important and under-researched. ${ }^{26}$ Additionally, it remains unclear if outward presenting phenotypes (eg, functional tremor) are driven by the same set of mechanisms across all patients or if there are a range of biological mechanisms that may lead to the same clinical phenotype. If neural mechanisms differ across patients with similar phenotypes, it will be important to understand if biologically informed subtypes are linked to specific treatment response and prognostic profiles. Such observations, if robustly elucidated, would facilitate the use of precision medicine in mFND care. Relatedly, research is needed to investigate if there are common neural mechanisms across FND and the spectrum of functional disorders across medicine. To 


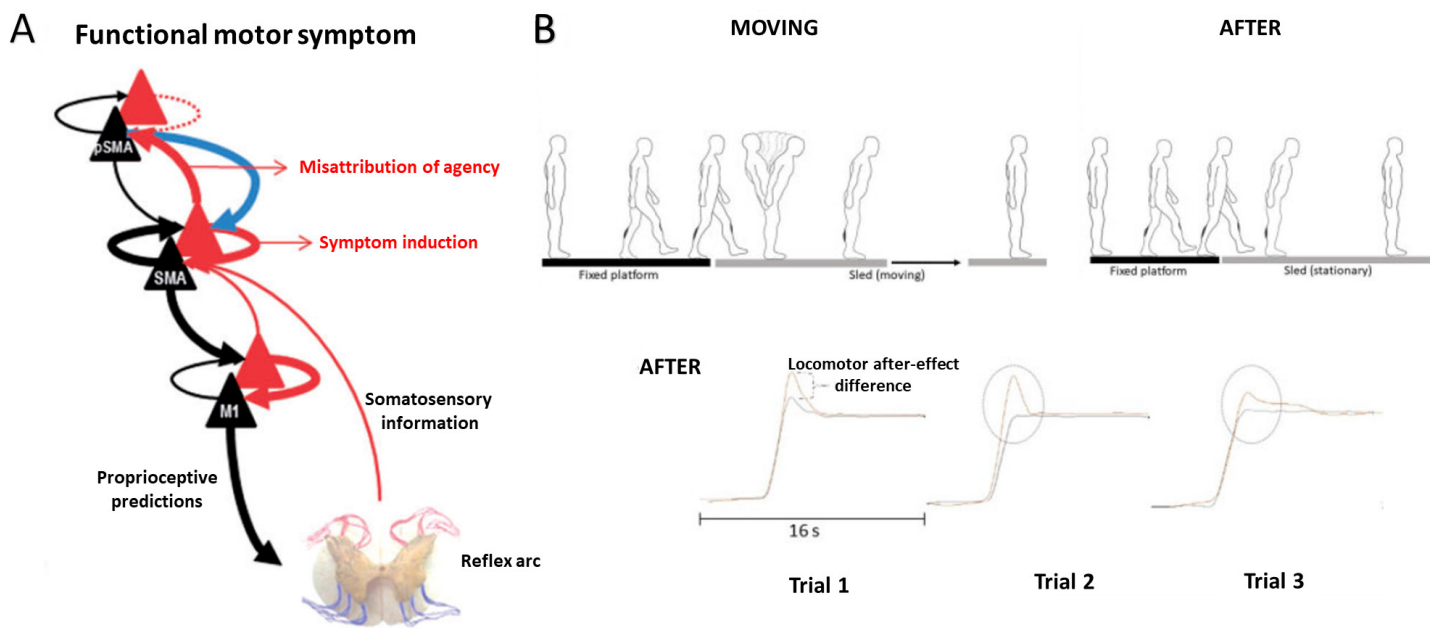

Figure 1 Model and evidence supporting the role for sensorimotor expectations and misdirected attention in the pathophysiology of motor functional neurological disorder. Based on Edwards et al, (A) shows the hierarchical anatomy that is theorised to underlie false inference in patients with functional motor symptoms. ${ }^{22}$ Within this model, abnormal prior expectation is formed within prediction units of intermediate motor areas (here the supplementary motor area (SMA)) (black triangles). This prior is afforded abnormal precision by attentional processes (blue arrow) that cause intermediate level motor predictions (thick black arrows) to elicit movement and prediction errors (thick red arrows) from prediction error units (red triangles) to report the unpredicted content of the movement to higher cortical areas (here, pre-SMA (pSMA)). The secondary consequence of these prediction errors is that prefrontal regions try to explain them in terms of symptomatic interpretation or misattribution of agency to external causes. Based on findings of Lin et al, (B) (top portion) displays the broken escalator phenomenon. ${ }^{23}$ Following a series of initial 'before' tasks where participants step onto and off of a stationary platform (not shown), participants then go on to step onto a moving sled performed 10 times ('moving'). In the 'after' trials, participants once again step onto a stationary sled five times. The 'broken escalator' phenomenon, also called the locomotor after effect, occurs when the learnt motor response during the 'moving' phase is carried forward in the 'after' trials. In (B) (lower panel), linear trunk displacement measurements show that only patients with a functional gait displayed persistence of the locomotor after effect across repeated 'after' trials. Lin et al suggested this reflected evidence of failed deadaptation (failure to update expectations).

comprehensively answer these questions, behavioural, electrophysiological, neuroimaging, autonomic, neuroendocrine and neuroinflammation data will likely be needed. Use of patient control groups, across co-occurring neurological, psychiatric and medical diagnoses, will also inform the specificity of neural mechanisms in mFND. ${ }^{\text {S18 }}$

\section{AETIOLOGICAL FACTORS}

\section{New developments}

Over the past decade, aetiological research in mFND contextualising predisposing vulnerabilities have identified the presence of a number of potential putative contributing factors, while at the same time, acknowledging the importance of individual differences. A systematic review and meta-analysis showed that the odds of being diagnosed with FND was 3.9 times higher given childhood physical abuse compared with controls and 3.3 times higher given childhood sexual abuse. ${ }^{27}$ In a separate systematic review examining the later-life consequences of childhood sexual abuse, the odds of experiencing sexual abuse was highest in FND compared with a range of mood, anxiety, personality and painrelated disorders. ${ }^{\mathrm{S} 19}$ Underscoring the importance of adverse life experiences is a study of 430 individuals with mixed FND identifying that nearly two-thirds reported active post-traumatic stress disorder (PTSD) symptoms. ${ }^{\mathrm{S} 2}$ Studies in mFND populations have also demonstrated the inter-relatedness between adverse life experiences and other predisposing vulnerabilities for the development of mFND, such as fearful attachment styles independently correlating with childhood abuse burden, alexithymia and depression scores. ${ }^{\text {S21 }}$ These findings highlight the importance of considering the relevance of adverse life experiences in mFND populations using stress-diathesis and neurodevelopmental perspectives, emphasising the interplay between biological (genetic/epigenetic) risk, life events and precipitating (triggering) factors. ${ }^{28} 29$ While in early stages, pathophysiology studies have started to contextualise the neurobiological importance of childhood maltreatment in promoting the development of mFND. ${ }^{24}$ S22 Two examples from the functional MRI literature include: (1) the observation that resting-state connectivity strength between salience/limbic network brain areas (amygdala, insula) and the precentral gyrus correlated with the magnitude of previously experienced childhood physical abuse in patients with $\mathrm{mFND}^{30}$ and (2) the finding that the G-703T polymorphism (rs4570625) in the tryptophan hydroxylase-2 (TPH2) gene moderated the relationship between childhood trauma and functional movement symptom severity; differential amygdalaprefrontal connectivity profiles were also identified in patients with mFND based on TPH2 genotype (see figure 2). ${ }^{31}$

Acknowledging that not all patients with mFND endorse adverse life experiences, risk factors for mFND extend beyond these considerations. A heightened bodily attentional focus, at times to the decrement of perceptual accuracy, has been characterised in patients with mFND. ${ }^{32}$ S23 Altered bodily attention and increased arousal may also help explain associations between physical injury and the subsequent development of $\mathrm{mFND},{ }^{33}$ given that physical injury promotes heightened attention to the self and activation of bodily arousal systems. S24 The traditional conceptualisation of several demographic and psychosocial factors has also been challenged, including the increased appreciation of mFND symptoms in older populations (eg, Parkinson's disease ${ }^{17}$ and findings that patients with mFND and neurological controls have similar histories of employment in healthcare related fields. ${ }^{\text {S25 }}$ Psychiatric diagnosis are common (eg, $1 / 3$ of patients with mFND meeting criteria for major depressive disorder), yet are not universally present 
A

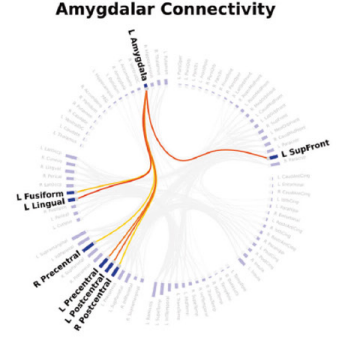

Insular Connectivity

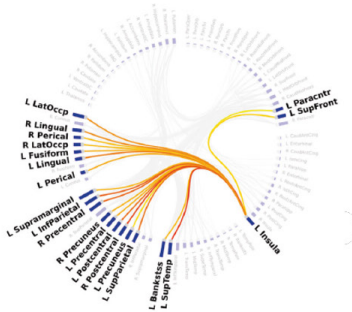

L Amygdala - L Precentral Gyrus

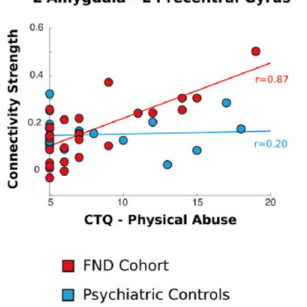

$\square$ Psychiatric Controls

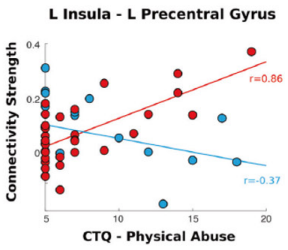

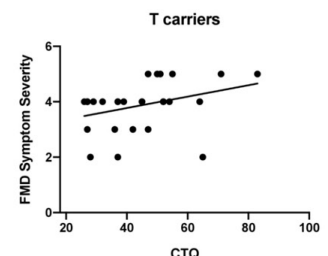

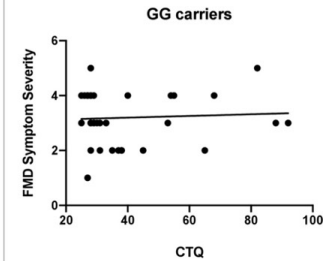

C

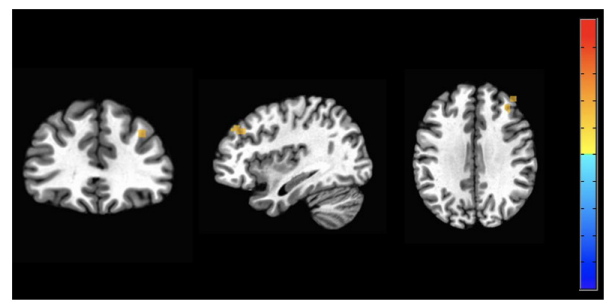

Figure 2 Examples of two recent functional neuroimaging studies bridging neural mechanisms and aetiological factors in motor functional neurological disorder (mFND). Based on the findings of Diez et al, (A) shows connectograms and scatterplots illustrating that resting-state functional connectivity strength between emotion processing brain areas (amygdala, insula) and primary motor cortex (precentral gyrus) positively correlated with the magnitude of previously experienced childhood physical abuse in patients with mixed mFND. ${ }^{30}$ Note: findings are bilateral but for ease of viewing only left hemisphere findings are displayed; self-reported childhood abuse burden was measured using the Childhood Trauma Questionnaire (CTQ). Similar childhood physical abuse-functional brain architecture relationships were not observed in psychiatric controls. Based on the findings of Spagnolo et al, (B) shows that childhood abuse burden correlated with functional movement disorder (FMD) symptom severity only in the subset of patients carrying the G-703T polymorphism (rs4570625) in the tryptophan hydroxylase-2 gene. (C) T carriers with mFND exhibited reduced right amygdala-middle frontal gyrus restingstate functional connectivity compared with GG homozygotes with mFND and healthy controls. ${ }^{31}$

in all patients with mFND. ${ }^{\text {S26 }}$ Trait psychological constructs remain important, such as the finding that alexithymia, independent of depression scores, was elevated in patients with mFND compared with neurological and healthy controls; patients with mFND and prominent alexithymia also exhibited higher rates of obsessive-compulsive personality disorder. ${ }^{34}$ Novel risk factors for the development of mFND have also been identified, such as aberrant sensory and information processing. ${ }^{35} 36$

\section{Implications}

Identification of a broad array of relevant, yet non-deterministic, risk factors for developing mFND suggests that links between aetiological factors and disease mechanism remain incompletely understood. More specifically, while adverse life experiences remain important vulnerabilities for developing mFND and are linked to other predisposing and perpetuating factors, the presence or absence of these events neither helps rule in nor rule out a diagnosis of mFND.

\section{Future directions}

Additional research is needed to understand the intersection of disease mechanisms, aetiological factors and treatment response within the context of the biopsychosocial framework (including spiritual and cultural influences). A precision medicine approach may be needed to not only link psychosocial risk factors to brain circuits but also to contextualise a range of relevant mediating and modulating factors including genetic/epigenetic information. The importance of developmental trajectories (including critical periods), gene-environment interactions and sex differences are also underexplored factors that may help better explain connections between risk factors and the later-life development of mFND. Given significant childhood maltreatment in a subset of patients with $\mathrm{mFND}$, future research may also inquire if there is a 'trauma subtype' of mFND, while also clarifying important risk factors in patients who lack such a history. The relevance of aetiological factors to treatment selection and response (eg, targeting concurrently present PTSD symptoms as an approach to treat $\mathrm{mFND}$ ) also requires more research inquiry.

\section{TREATMENTS AND PROGNOSIS \\ New developments}

The late 20th century's lack of interest and investment in mFND by healthcare systems is reflected in exceedingly few care programmes for this population, which in turn is reflected in patients feeling marginalised and unable to access treatments. ${ }^{27}$ S28

With the DSM-5 modifications, the neurologist's (and other clinician's) role in mFND has now expanded to making a positive 'rule-in' diagnosis, communicating the diagnosis effectively and facilitating access to additional treatments. 'How to' articles have disseminated good clinical practices on the delivery of the diagnosis and longitudinal care. ${ }^{18}$ S29 Specialist FND clinics, often led jointly by neurologists and psychiatrists, have also been developed in some countries for complex cases (eg, those with

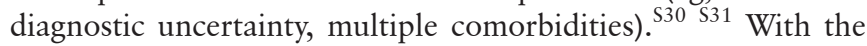
time required to adequately manage this population, challenges have been raised regarding clinical bandwidth. ${ }^{\mathrm{S} 30}$

The website neurosymptoms.org has become a valuable educational resource for patients and clinicians. Other information websites have been created, including from patient support charities (eg, fndhope.org, fndaction.org.uk). The efficacy of online information and self-help used in isolation was assessed in a randomised controlled trial (RCT). ${ }^{37}$ At 3 months, there was no difference in improvement on self-rated health or in secondary outcomes between groups. This suggests that online education, while generally rated favourably, is inadequate as a stand-alone treatment. 
Table 2 Randomised controlled trials in motor functional neurological disorder over the past decade

\begin{tabular}{lll}
\hline Study & $\mathbf{n}$ & Description \\
\hline Physiotherapy & & \\
Nielsen et $\left.\right|^{40}$ & 60 & Randomised feasibility study of specialist physiotherapy versus usual \\
& care \\
& Duration and setting: 5 days in an intensive outpatient/day \\
& programme \\
& Outcome measures: Feasibility (recruitment and retention rates, \\
& intervention fidelity, acceptability); SF-36; WSAS; EQ-5D-5L; DASH; \\
& CGI-patient rated; FMS; BBS
\end{tabular}

Points

\section{Multidisciplinary rehabilitation} Jordbru et $a^{39}$
60
Randomised study of multidisciplinary rehabilitation versus a wait list control for functional gait disorder

Duration and setting: 3-week inpatient programme

Outcome measures: FIM, FMS, SF-12

The intervention was delivered by physiotherapists and included education movement retraining and self-management. The control was standard community neurophysiotherapy.

At 6-month follow-up, $72 \%$ of the intervention group reported symptom improvement, compared with $18 \%$ of the controls. Significant improvement was seen in a range of physical and quality-of-life outcome measures. The intervention was associated with a gain in quality adjusted life years and an incremental cost effectiveness ratio that was suggestive of a cost-effective intervention.

This is the first and currently only randomised study of multidisciplinary rehabilitation (described by the authors as 'adapted physical activity with a cognitive behavioural framework').

Post treatment, there was a significant difference between groups in physical and quality-of-life outcome assessments. Treatment gains were, for the most part, maintained at 12-month follow-up.

Cognitive behavioural therapy

Dallocchio et $a l^{42}$

29 A pilot, single-blinded randomised study, comparing CBT alone versus CBT plus physical activity versus SMC

Duration and setting: 12 weeks of outpatient 90 -minute CBT with or without adjunctive outpatient physical activity $(60 \mathrm{~min}$, two times per week)

Outcome measures: PMDRS; PHQ-15

\begin{tabular}{|c|c|c|}
\hline Sharpe et $a l^{43}$ & $127^{*}$ & $\begin{array}{l}\text { Randomised controlled trial of CBT-based guided self-help plus usual } \\
\text { care versus usual care alone } \\
\text { Duration and setting: self-guided outpatient CBT plus four 30-minute } \\
\text { face-to-face guidance sessions over } 3 \text { months } \\
\text { Outcome measures: CGI-patient rated; SF-12; PHQ-13 }\end{array}$ \\
\hline
\end{tabular}

Participants allocated to self-help CBT reported greater improvement on the primary outcome of self-rated health (CGI). At 6 months, the treatment effect was no longer statistically significant.

The two CBT containing interventions (with and without physica activity) showed reduction in PMDRS over 12 weeks. SMC showed no improvements

\section{Botulinum neurotoxin}

Dreissen et al ${ }^{44} 49$

Online education and self-help

Gelauff et $a \beta^{37} \quad 186$

Randomised, double-blinded controlled trial of botulinum neurotoxin versus placebo (sterile saline) injections

Duration and setting: two outpatient injections 3 months apart,

followed by a 10-month open-label extension

Outcome measures: CGI-clinician and CGI-patient rated; PMDRS; SF-

36; AMC Linear Disability Score

Randomised controlled trial of botulinum neurotoxin versus placebo

(sterile saline) injections, followed by CBT

Duration and setting: outpatient injection followed by 12 weeks of

psychotherapy

Outcome measures: PMDRS, Katz Index of Independence in ADLs,

Lawton instrumental ADL
At 4 months, there were no statistically significant differences between treatment arms across primary and secondary outcomes. However, improvement was observed across both treatment arms (56\%-64\%), suggesting a notable placebo effect. Across the length of the entire trial, $81 \%$ improved from baseline.

There were no differences in clinical outcomes at 12 weeks. While both treatment arms showed a tendency toward improvement, a statistically significant change from baseline was only observed in the placebo+CBT group.
There has been a rise in physical rehabilitation and multidisciplinary research. ${ }^{38}$ Since 2010 , no less than 17 rehabilitation cohort studies of patients with mFND have been published (2 RCTs, 7 prospective and 8 retrospective). The first RCT, published in 2014, compared a 3-week inpatient multidisciplinary rehabilitation programme to a waiting list control, for patients with functional gait disorders. ${ }^{39}$ Improvements were seen in the treatment arm in measures of physical health that were maintained at 12-month follow-up. The second RCT was a feasibility study of specialist physiotherapy, comprising psychologically informed education and movement retraining. ${ }^{40}$ Sixty patients were randomised to the intervention versus standard community neurophysiotherapy, with $72 \%$ of the intervention participants reporting motor improvements compared with $18 \%$ of controls at 6-month follow-up. See table 2 for additional details and supporting evidence. ${ }^{\$ 32-43}$

A factor complicating synthesis of the evidence for physiotherapy and multidisciplinary rehabilitation is heterogeneity in the interventions. Differences include treatment setting (outpatient, inpatient, day hospital), medical specialty involved (psychiatry, neurology, physical rehabilitation, etc), treatment duration/intensity and type of therapy and combination of modalities (physiotherapy, cognitive behavioural therapy (CBT), psychoeducation, movement retraining, non-specific exercise, etc). Despite differences, common elements across treatments include starting with a diagnostic explanation based on a patientcentred biopsychosocial model. Motor symptoms are often conceptualised as a disconnect between the brain and body, and 
self-directed attention is usually emphasised as a factor exacerbating symptoms. Physical therapies aim to retrain movement with diverted attention, and physical interventions are informed by a psychological understanding of symptoms (eg, addressing fear-avoidance behaviours using graded exposure).

As well summarised in a recent systematic review, the major advance for psychotherapy in mFND is that initial RCTs have been conducted and published - after a dearth of controlled data in decades prior. ${ }^{41}$ Examples of the interventions are described below and summarised in table 2 and online supplemental table 1.

Psychotherapy trials for mFND include a pilot single-blind RCT of 29 patients with mFND (mostly functional tremor) randomised to receive 12 weeks of conventional CBT alone (90-minute session, once a week) versus CBT+adjunctive physical activity (APA) (60-minute session, two times per week of low-intensity/moderate-intensity walking). ${ }^{42}$ The control group consisted of eight patients receiving standard medical care (SMC). The CBT intervention focused on the interplay of somatic misinterpretations, negative thoughts, illness beliefs and low mood or anxiety, along with use of distraction, relaxation and other problem-solving techniques. The two CBT containing interventions (with and without APA) showed improvements in functional motor symptoms, depression and anxiety scores at 12 weeks, while the SMC arm showed no significant improvements. A prospective single-arm study in 15 patients with functional tremor also demonstrated the efficacy for CBT in reducing tremor severity. ${ }^{26}$ Furthermore, an RCT of self-guided CBT in 127 patients with mixed FND randomised to CBT+usualcare $(n=64)$ versus usual care alone $(n=63)$ showed a statistically significant improvement in patient-rated global improvement at 3 months for those receiving CBT; reductions in somatic symptom burden and health anxiety were also observed. These gains were no longer significant at 6-month follow-up. ${ }^{43}$ See online supplemental table 1 for details regarding two small psychodynamic psychotherapy RCTs, as well as other psychotherapy cohort studies in mFND populations. ${ }^{\text {S44-46 }}$

Regarding paediatric mFND-while there are ethical and practical challenges to performing RCTs in this populationefficacious multidisciplinary programmes generally combine psychotherapy, physiotherapy, occupational therapy and family work targeting focus of attention and pertinent stressors and school attendance/reintegration (see online supplemental table 1). 5 S47-S49

There remains little evidence for pharmacological therapy in the direct treatment of mFND symptoms, yet medications have a role in managing concurrently present anxiety, depression, migraine and insomnia. Regarding other treatments, a recent randomised placebo-controlled trial of botulinum neurotoxin (BoNT) for jerky and tremulous functional movement disorder $(n=48)$ found no benefit compared with placebo. ${ }^{44}$ Here, approximately two-thirds of patients in both groups improved, demonstrating a large placebo effect. A similar positive placebo response was observed in a pilot randomised trial of BoNT followed by 12 weeks of CBT in patients with functional dystonia $(n=14) .{ }^{45}$ While placebo effects are important considerations, ${ }^{S 50}$ there is an argument to be made for the use of BoNT in patients with chronic symptoms that have not benefited from other treatments. Transcranial magnetic stimulation (TMS) also continues to be investigated as a promising therapeutic, although disentangling circuit-level neuromodulatory effects from placebo remains challenging. ${ }^{551}$ When placebo is considered the 'active ingredient', there remains debate regarding how transparent to be with patients (we favour an open and transparent stance).
Given that non-specialist clinicians may feel ill prepared to assess and manage patients with $\mathrm{mFND},{ }^{\mathrm{S} 52}$ expert opinion-based recommendations and practical advice are welcomed additions. These include:

- Assessment and diagnosis of mFND symptoms. ${ }^{46}$

- Neuropsychiatric assessment. ${ }^{\mathrm{S} 3}$

- Delivering the diagnosis (including providing clear, empathic communication with a cautiously optimistic stance for improvement). ${ }^{18}$

- mFND presenting to stroke services. ${ }^{553}$

- Physiotherapy. $^{47}$

- Occupational therapy. ${ }^{48}$

Consensus recommendations to standardise outcome measures for clinical trial research in FND have also been published, emphasising patient-reported data. ${ }^{49}$

Regarding prognosis, a systemic review of long-term follow-up studies from 10 to 491 individuals reported that $39 \%$ of patients across the spectrum of FND were the same or worse and the majority (approximately 80\%) remained symptomatic. ${ }^{\text {S54 }}$ The same research group recently published a 14-year follow-up study in 76 adults with weakness, identifying that $20 \%$ had symptom resolution, 31\% improved, 23\% were the same and 26\% were worse. ${ }^{16}$ In terms of discrete prognostic factors in adults, findings have been inconsistent and understudied in more recently developed care models. Outcomes from specialist paediatric multidisciplinary programmes are more optimistic with approximately three quarters of children returning to full health and full-time school attendance. ${ }^{5}$ Outcomes are less favourable for children with chronic mFND symptoms at presentation; those with cognitive vulnerabilities, whose comorbid mental health disorders or other (comorbid) functional somatic symptoms do not resolve and those who subsequently develop chronic mental health problems.

\section{Implications}

While delivery of the diagnosis is the first step in treatment, online self-help information alone is insufficient for symptom reduction and should not be considered definitive treatment. Likewise, self-help psychotherapy approaches appear to lack durability in maintaining improvement. Careful assessment is needed to triage patients towards the most suitable treatment based on available options, including physiotherapy, skills based psychotherapy and/or multidisciplinary interventions. Given evolving care models and lack of robust predictors of prognosis, those with chronic symptoms, formerly considered refractory, should not be excluded from evidence-based treatments.

\section{Future directions}

To further advance mFND treatments, future research should continue to pursue fully powered RCTs across rehabilitative and psychological interventions. Studies examining optimal treatment setting(s) are also needed.

An important future direction could be to develop specific interventions that are tailored both towards the mFND phenotype (eg, weakness, tremor, dystonia) and the wider clinical syndrome. For example, in addition to motor symptoms, common comorbidities and other health-related problems could be considered within a single-treatment package (eg, PTSD, anxiety, chronic pain, migraine, joint hypermobility, social difficulties, etc). The timing of the different treatment elements may also be important, and the value of a modular approach to treatment could be explored, where the focus of whole-person treatment can be personalised and evolve according to the patient's 
biopsychosocial clinical formulation. As an example, a patient with a functional gait disorder and major depression with suicidality may benefit from physical therapy at some point, but it is probably more important initially to treat aggressively their depression and existential concerns. Conversely, a patient with sudden onset disabling physical symptoms may need to make some initial progress with physical therapy (eg, to regain sitting balance) before engaging well in psychotherapy.

Additional research should explore the development of techrehabilitation adjuncts (informed by advances in elucidating mFND pathophysiology) and innovations to improve access to specialist treatment (eg, tele/remote health as has been used in other FND subtypes, ${ }^{50}$ virtual reality, wearable technology, biofeedback, TMS, etc). More aggressive focus on psychosocial factors might also be useful. Additional work is needed to define the most suitable clinical outcome measures and to also determine if the creation of new FND-specific outcome measures may be beneficial. Further clarifying neural mechanisms and predictors of treatment response will also be important, ${ }^{26}$ offering the potential to develop novel psychologically and biologically informed treatment interventions. Finally, it is crucial that more treatment programmes are developed; it will not do any good to find optimal treatment strategies if they will not be available to the majority of patients.

\section{STIGMA}

\section{New developments}

Stigma pertaining to the diagnosis of $\mathrm{mFND}$ is increasingly recognised as an important, multifaceted issue requiring clinical and research attention. In mFND, stigma represents a complex interplay between patients, clinician-patient relationships, healthcare systems and sociocultural factors. The very fact that mFND sits at the intersection of neurology and psychiatry challenges deeply rooted medical and societal norms of health and disease. Furthermore, the variability/distractibility seen in many individuals mistakenly perpetuates a framing that symptoms are voluntary (when perceived nonetheless as involuntary by the patient). These and other nuanced issues related to stigma can be discussed across three levels-public stigma, personal selfstigma and patient label avoidance-and we use these categories to frame our discussion (see box 1). ${ }^{5152}$

\section{Box 1 Three different aspects of stigma}

Stigma category and definition

- Public stigma occurs when the general population-or certain subsets of the population-endorses negative beliefs pertaining to a certain illness and acts on these beliefs in a discriminatory manner, often by avoidance and withdrawal.

- Personal stigma occurs when the individual person-child or adult or the family - becomes aware of the negative beliefs about a certain illness, internalises these beliefs and applies them to the self.

- Patient label avoidance refers to the patient's reluctance and efforts to distance himself or herself from a label-in the case of motor functional neurological disorder, a diagnosisbecause the label is perceived as being socially unacceptable. This type of stigma is more commonly perceived in relation to mental health disorders, because such disorders are commonly misperceived as being the result of personal weakness or poor character.
With the growth of scientifically based medicine and the complexities in how to understand functional neurological symptoms, mFND was sidelined and publicly stigmatised into the category of 'medically unexplained' disorders in the late 20th century. Interest in mFND waned reflected in mFND largely disappearing from medical textbooks, educational curricula and bedside teaching. ${ }^{\mathrm{S} 1}$ Relatedly, many physicians avoided the diagnosis-or used terminology that was offensive (eg, confusing mFND with malingering)-leaving patients perplexed or angry and setting up a negative process whereby patients sought help from multiple physicians, were subjected to repeated unnecessary diagnostic tests and treatment was delayed or not provided. ${ }^{18}$

Public stigma regarding $\mathrm{mFND}$ has unfortunately continued. ${ }^{555}$ Some physicians caring for patients with $\mathrm{mFND}$ may manifest their discomfort in non-verbal communication patterns that convey uncertainty, negative treatment expectations and/or their own perceptions that the diagnosis is a delicate (stigmatised) matter that needs to be managed cautiously (or at least not by a neurologist). ${ }^{556}$ In doing so, physicians undermine their own capacity to use positive suggestion to influence belief-relevant in the treatment of mFND-and core to the art of healing in all of medicine. ${ }^{553}$ Patients can also have negative interactions with other healthcare professionals (eg, nurses, administrative staff), coworkers, friends and family - driven in part by the conceptual misunderstandings outlined above. The general lack of dedicated services in hospitals and the failure to include FND in national research priorities also communicate a powerful message that mFND is seemingly unimportant.

Patients with mFND may internalise stigma, make it personal and report being harmed by it. ${ }^{556}$ Internalisation of stigma promotes feelings of vulnerability, helplessness, hopelessness, frustration and anger. ${ }^{52}$ Stigma contributes to the shaping of patients' own internal beliefs and expectations, which may limit their ability to improve. ${ }^{5}$ These factors also contribute to negative doctor-patient interactions. ${ }^{556}$

As a consequence, some patients find it difficult to accept an mFND diagnosis and others reject the diagnosis altogether or litigate the physician. ${ }^{556557}$ This label avoidance contributes both to clinician anxiety and potentially to endless doctor shopping (and medical procedures) in hopes of receiving a different 'medical' diagnosis. Sometimes label avoidance even propels patients to accept explanations that are outlandish or that conceptualise their $\mathrm{mFND}$ as a 'medical mystery'. ${ }^{57} \$ 58$

Changes in how the diagnosis is communicated to patients are occurring, including more clinicians using the term 'functional'. Educational efforts are underway to help physicians avoid the pitfalls of oversimplified explanations to patients that 'it is all stress-related' and moving towards describing 'stressors' in the context of life events. ${ }^{4} 7$ The multidisciplinary FND Society, several authoritative FND textbooks, growth of specialised treatment programmes and high impact publications advocating for change and research funding indicate that $\mathrm{mFND}$ is re-entering mainstream medicine. ${ }^{245} \$ 30 \$ 31 \$ 59$

\section{Implications}

Public stigma, personal self-stigma and patient label avoidance in mFND remain major concerns, and efforts to mitigate stigma need to be driven by clinicians, researchers, patients, advocacy groups and policy-makers. A helpful transition that has occurred in recent years is the framing of mFND at the intersection of neurology and psychiatry (synonymous with a core neuropsychiatric disorder), and rigorous pathophysiology research in mFND is also decreasing stigma by advancing a brain-based, mechanistic 
understanding of the condition. Unfortunately, mental health is stigmatised in many societies and healthcare systems in comparison to medical/neurological conditions. This is a notable issue, given that the multidisciplinary approach to mFND patient care includes important roles for mental health clinicians. ${ }^{3}$ Treatment implications that emerge from this body of work highlight the importance of the biopsychosocial model-the body, mind and family and social context - to address each patient's particular presentation and issues. The mFND field needs to be cautious so as to strike a good balance between neurological and psychological/psychiatric conceptualisations of the disorder. Moreover, mental health professionals need to be empowered at the same level of engagement as neurologists, rehabilitation specialists and research scientists, as we work collaboratively for the benefit of patients.

\section{Future directions}

In addition to increasing advocacy to further decrease stigma pertaining to mFND and related conditions across medicine and society, mFND can serve as a model condition through which to challenge the inherently artificial divide between physical and mental health that is pervasive in medicine and society-a dualism exposed most directly by mFND. This condition also offers the potential to bring the disciplines of neurology and psychiatry, two specialties for the same organ system, increasingly together to leverage an integrated clinical neuroscience perspective.

\section{CONCLUSIONS}

Significant advances have occurred in the past decade in the diagnosis, conceptual understanding and treatment of mFND. This progress reflects a renaissance taking place across the field of FND, bringing neurology and psychiatry together again, as we are confronted by the need for multidisciplinary and collaborative care models for this complex population. With a biopsychosocial understanding of $\mathrm{mFND}$ and with better neuroscientific tools to provide rigorous biological phenotyping and classification, we are poised to design well-constructed and patientcentred clinical trials to benefit patients with this condition.

Contributors All authors contributed to the planning, literature review, drafting and editing of the manuscript.

Funding DLP was funded by the National Institute of Mental Health (NIMH) Grant K23MH111983-04 and the Sidney R. Baer Jr. Foundation. MH was supported by the National Institute of Neurological Disorders and Stroke (NINDS) Intramural Programme. GN receives research funding from the National Institute for Health Research.

Competing interests DLP has received honoraria for continuing medical education lectures in functional neurological disorder and is on the editorial board of Epilepsy \& Behavior. MH is an inventor of patents held by National Institutes of Health $(\mathrm{NIH})$ for an immunotoxin for the treatment of focal movement disorders and the $\mathrm{H}$-coil for magnetic stimulation; in relation to the latter, he has received license fee payments from the NIH (from Brainsway). He is on the medical advisory boards of Cala Health and Brainsway. He has research grants from Allergan for studies of methods to inject botulinum toxins, Medtronic, Inc. for a study of deep brain stimulation (DBS) for dystonia and Cala Health for studies of a device to suppress tremor. WCL receives editor's royalties from the publication of Gates and Rowan's Nonepileptic Seizures, 3rd edition (Cambridge University Press, 2010) and 4th edition (2018) and author's royalties for Taking Control of Your Seizures: Workbook and Therapist Guide (Oxford University Press, 2015) and receives research support from the Department of Defense (DoD W81XWH-17-0169).

Patient consent for publication Not required.

Provenance and peer review Commissioned; externally peer reviewed.

Supplemental material This content has been supplied by the author(s). It has not been vetted by BMJ Publishing Group Limited (BMJ) and may not have been peer-reviewed. Any opinions or recommendations discussed are solely those of the author(s) and are not endorsed by BMJ. BMJ disclaims all liability and responsibility arising from any reliance placed on the content. Where the content includes any translated material, BMJ does not warrant the accuracy and reliability of the translations (including but not limited to local regulations, clinical guidelines, terminology, drug names and drug dosages), and is not responsible for any error and/or omissions arising from translation and adaptation or otherwise.

\section{ORCID iDs}

David L Perez http://orcid.org/0000-0003-2721-583X

Mark Hallett http://orcid.org/0000-0002-3180-6811

W Curt LaFrance, Jr http://orcid.org/0000-0002-4901-3852

\section{REFERENCES}

1 Espay AJ, Aybek S, Carson A, et al. Current concepts in diagnosis and treatment of functional neurological disorders. JAMA Neurol 2018;75:1132-41.

2 Stephen $\mathrm{CD}$, Fung $\mathrm{V}$, Lungu $\mathrm{Cl}$, et al. Assessment of emergency department and inpatient use and costs in adult and pediatric functional neurological disorders. JAMA Neurol 2021;78:88-101.

3 Pick S, Goldstein LH, Perez DL, et al. Emotional processing in functional neurological disorder: a review, biopsychosocial model and research agenda. J Neurol Neurosurg Psychiatry 2019;90:704-11.

4 Hallett M, Stone J, Carson AJ. Functional neurologic disorders. Amsterdam: Elsevier, 2016.

5 Kozlowska K, Scher S, Helgeland H. Functional somatic symptoms in children and adolescents: the Stress-System approach to assessment and treatment. London: Palgrave Macmillan, 2020.

6 Daum C, Hubschmid M, Aybek S. The value of 'positive' clinical signs for weakness, sensory and gait disorders in conversion disorder: a systematic and narrative review. J Neurol Neurosurg Psychiatry 2014;85:180-90.

7 LaFaver K, Lang AE, Stone J, et al. Opinions and clinical practices related to diagnosing and managing functional (psychogenic) movement disorders: changes in the last decade. Eur J Neurol 2020;27:975-84.

8 Schwingenschuh P, Katschnig P, Seiler S, et al. Moving toward "laboratory-supported" criteria for psychogenic tremor. Mov Disord 2011;26:2509-15.

9 Daum C, Gheorghita F, Spatola M, et al. Interobserver agreement and validity of bedside 'positive signs' for functional weakness, sensory and gait disorders in conversion disorder: a pilot study. J Neurol Neurosurg Psychiatry 2015;86:425-30.

10 Espay AJ, Lang AE. Phenotype-specific diagnosis of functional (psychogenic) movement disorders. Curr Neurol Neurosci Rep 2015;15:32.

11 van der Salm SMA, Tijssen MAJ, Koelman JHTM, et al. The Bereitschaftspotential in jerky movement disorders. J Neurol Neurosurg Psychiatry 2012;83:1162-7.

12 Ganos C, Martino D, Espay AJ, et al. Tics and functional tic-like movements: can we tell them apart? Neurology 2019;93:750-8.

13 Nonnekes J, Růžička E, Serranová T, et al. Functional gait disorders: a sign-based approach. Neurology 2020;94:1093-9.

14 Hawley JS, Weiner WJ. Psychogenic dystonia and peripheral trauma. Neurology 2011;77:496-502.

15 Kaski D, Bronstein AM, Edwards MJ, et al. Cranial functional (psychogenic) movement disorders. Lancet Neurol 2015;14:1196-205.

16 Gelauff JM, Carson A, Ludwig L, et al. The prognosis of functional limb weakness: a 14-year case-control study. Brain 2019:142:2137-48.

17 Wissel BD, Dwivedi AK, Merola A, et al. Functional neurological disorders in Parkinson disease. J Neurol Neurosurg Psychiatry 2018;89:566-71.

18 Carson A, Lehn A, Ludwig L, et al. Explaining functional disorders in the neurology clinic: a photo story. Pract Neurol 2016;16:56-61.

19 Stone J, Edwards M. Trick or treat? showing patients with functional (psychogenic) motor symptoms their physical signs. Neurology 2012;79:282-4.

20 Schwingenschuh P, Katschnig P, Edwards MJ, et al. The blink reflex recovery cycle differs between essential and presumed psychogenic blepharospasm. Neurology 2011;76:610-4.

21 Galli S, Tatu L, Bogousslavsky J, et al. Conversion, factitious disorder and malingering: a distinct pattern or a continuum? Front Neurol Neurosci 2018:42:72-80.

22 Edwards MJ, Adams RA, Brown H, et al. A Bayesian account of 'hysteria'. Brain 2012;135:3495-512

23 Lin D, Castro P, Edwards A, et al. Dissociated motor learning and de-adaptation in patients with functional gait disorders. Brain 2020;143:2594-606.

24 Maurer CW, LaFaver K, Ameli R, et al. Impaired self-agency in functional movement disorders: a resting-state fMRI study. Neurology 2016;87:564-70.

25 Aybek S, Nicholson TR, Zelaya F, et al. Neural correlates of recall of life events in conversion disorder. JAMA Psychiatry 2014;71:52-60.

26 Espay AJ, Ries S, Maloney T, et al. Clinical and neural responses to cognitive behavioral therapy for functional tremor. Neurology 2019;93:e1787-98.

27 Ludwig L, Pasman JA, Nicholson T, et al. Stressful life events and maltreatment in conversion (functional neurological) disorder: systematic review and meta-analysis of case-control studies. Lancet Psychiatry 2018;5:307-20.

28 Keynejad RC, Frodl T, Kanaan R, et al. Stress and functional neurological disorders: mechanistic insights. J Neurol Neurosurg Psychiatry 2019;90:813-21. 
29 Apazoglou K, Adouan W, Aubry J-M, et al. Increased methylation of the oxytocin receptor gene in motor functional neurological disorder: a preliminary study. J Neurol Neurosurg Psychiatry 2018;89:552-4.

30 Diez I, Larson AG, Nakhate V, et al. Early-Life trauma endophenotypes and brain circuit-gene expression relationships in functional neurological (conversion) disorder. Mol Psychiatry 2020. doi:10.1038/s41380-020-0665-0. [Epub ahead of print: 12 Feb 2020].

31 Spagnolo PA, Norato G, Maurer CW, et al. Effects of TPH2 gene variation and childhood trauma on the clinical and circuit-level phenotype of functional movement disorders. J Neurol Neurosurg Psychiatry 2020;91:814-21.

32 Pareés I, Saifee TA, Kassavetis P, et al. Believing is perceiving: mismatch between selfreport and actigraphy in psychogenic tremor. Brain 2012;135:117-23.

33 Kozlowska K, Palmer DM, Brown KJ, et al. Reduction of autonomic regulation in children and adolescents with conversion disorders. Psychosom Med 2015;77:356-70.

34 Demartini B, Petrochilos P, Ricciardi L, et al. The role of alexithymia in the development of functional motor symptoms (conversion disorder). J Neurol Neurosurg Psychiatry 2014;85:1132-7.

35 Sadnicka A, Daum C, Meppelink A-M, et al. Reduced drift rate: a biomarker of impaired information processing in functional movement disorders. Brain 2020;143:674-83

36 Pareés I, Kassavetis P, Saifee TA, et al. 'Jumping to conclusions' bias in functional movement disorders. J Neurol Neurosurg Psychiatry 2012;83:460-3.

37 Gelauff JM, Rosmalen JGM, Carson A, et al. Internet-based self-help randomized trial for motor functional neurologic disorder (shift). Neurology 2020;95:e1883-96.

38 Nielsen G, Stone J, Edwards MJ. Physiotherapy for functional (psychogenic) motor symptoms: a systematic review. J Psychosom Res 2013;75:93-102.

39 Jordbru AA, Smedstad LM, Klungsøyr 0, et al. Psychogenic gait disorder: a randomized controlled trial of physical rehabilitation with one-year follow-up. J Rehabil Med 2014;46:181-7.

40 Nielsen G, Buszewicz M, Stevenson F, et al. Randomised feasibility study of physiotherapy for patients with functional motor symptoms. J Neurol Neurosurg Psychiatry 2017;88:484-90.
41 Gutkin M, McLean L, Brown R, et al. Systematic review of psychotherapy for adults with functional neurological disorder. J Neuro/ Neurosurg Psychiatry 202010.1136/ jnnp-2019-321926. [Epub ahead of print: 05 Nov 2020].

42 Dallocchio C, Tinazzi M, Bombieri F, et al. Cognitive behavioural therapy and adjunctive physical activity for functional movement disorders (conversion disorder): a pilot, single-blinded, randomized study. Psychother Psychosom 2016;85:381-3.

43 Sharpe M, Walker J, Williams C, et al. Guided self-help for functional (psychogenic) symptoms: a randomized controlled efficacy trial. Neurology 2011;77:564-72.

44 Dreissen YEM, Dijk JM, Gelauff JM, et al. Botulinum neurotoxin treatment in jerky and tremulous functional movement disorders: a double-blind, randomised placebo-controlled trial with an open-label extension. J Neurol Neurosurg Psychiatry 2019:90:1244-50.

45 Vizcarra JA, Lopez-Castellanos JR, Dwivedi AK, et al. OnabotulinumtoxinA and cognitive behavioral therapy in functional dystonia: a pilot randomized clinical trial. Parkinsonism Relat Disord 2019:63:174-8.

46 Stone J. Functional neurological disorders: the neurological assessment as treatment. Pract Neurol 2016;16:7-17.

47 Nielsen G, Stone J, Matthews A, et al. Physiotherapy for functional motor disorders: a consensus recommendation. J Neurol Neurosurg Psychiatry 2015;86:1113-9.

48 Nicholson C, Edwards MJ, Carson AJ, et al. Occupational therapy consensus recommendations for functional neurological disorder. J Neurol Neurosurg Psychiatry 2020;91:1037-45.

49 Pick S, Anderson DG, Asadi-Pooya AA, et al. Outcome measurement in functional neurological disorder: a systematic review and recommendations. I Neurol Neurosurg Psychiatry 2020;91:638-49.

50 LaFrance WC Jr, Ho WLN, Bhatla A, et al. Treatment of psychogenic nonepileptic seizures (PNES) using video telehealth. Epilepsia 2020;61:2572-82.

51 Corrigan PW. Lessons learned from unintended consequences about erasing the stigma of mental illness. World Psychiatry 2016;15:67-73.

52 Rommelfanger $\mathrm{KS}$, Factor SA, LaRoche $\mathrm{S}$, et al. Disentangling stigma from functional neurological disorders: conference report and roadmap for the future. Front Neurol 2017:8:106.

53 Wieder L, Brown R, Thompson T, et al. Suggestibility in functional neurological disorder: a meta-analysis. J Neurol Neurosurg Psychiatry 2021;92:150-7. 